\title{
La cultura organizacional y la asociatividad empresarial de las microempresas del sector de confecciones textiles del distrito de Tacna, período 2012.
}

\author{
Organizational culture and micro business partnership of apparel textile industry of district Tacna,
} period 2012.

Renzi Marilu Loza Ticona ${ }^{1}$

\section{RESUMEN}

Objetivo: Determinar la relación que existe entre la cultura organizacional y la asociatividad empresarial de las microempresas del sector confecciones textiles del distrito de Tacna, período 2012.

Método: La investigación corresponde al tipo básica, porque tiene como finalidad la obtención y recopilación de información para ir construyendo una base de conocimiento que se va agregando a la información previa existente.

Resultado: Se analizaron e interpretaron la información procesada a través del análisis cuantitativo, cualitativo, y la síntesis, determinándose la relación que existe entre la cultura organizacional y la asociatividad empresarial de las microempresas del sector confecciones textiles del distrito de Tacna, período 2012.

Conclusiones: Existe relación significativa entre la cultura organizacional y la asociatividad empresarial de las microempresas del sector confecciones textiles del distrito de Tacna, período 2012.

La cultura organizacional que presentan las microempresas del Sector Confecciones Textiles del distrito de Tacna, es inadecuada.

Existe relación significativa baja entre la cultura organizacional y la asociatividad empresarial de las microempresas del sector confecciones textiles del distrito de Tacna, período 2012.

Finalmente, la asociatividad empresarial que presentan las microempresas del Sector Confecciones Textiles del distrito de Tacna es deficiente, 2012.

\section{PALABRA CLAVE}

Cultura Organizacional, asociatividad empresarial, sector confecciones textiles, énfasis en el equipo, tolerancia al conflicto, orientación hacia los resultados.

\section{ABSTRACT}

Objective: To determine the relationship between organizational culture and business associations of micro textile garment district of Tacna sector 2012 period.

Method: The research is for the basic type, because it has the purpose of obtaining and gathering information in order to build a knowledge base to be added to the existing background information.

Result: Were analyzed and interpreted the data processed through quantitative, qualitative analysis, and synthesis, determining the relationship between organizational culture and business association of microenterprises in the textile manufacturing industry Tacna District, 2012 period.

Conclusions: There is significant relationship between organizational culture and business association of microenterprises in the textile manufacturing industry Tacna District, 2012 period.

The organizational culture present micro Textile Garments Sector Tacna district is inadequate.

There is significant relationship between low organizational culture and business association of microenterprises in the textile manufacturing industry Tacna District, 2012 period.

Finally, business associations presenting micro Textile Garments Sector Tacn a district is poor, 2012.

\section{KEYWORDS}

Organizational culture, business associations, textile apparel sector, emphasis on the team, conflict tolerance, results orientation..

1. Doctora en Administración UPT, Magister en Administración y Dirección de Empresas UPT, Lic. en Adm. UNJBG, Ing. Comercial UJCM. Actualmente labora en la Universidad José Carlos Mariátegui de Moquegua, como docente de los cursos de Metodología de Investigación Científica y Taller de Tesis. 


\section{Introducción}

En el Sector confecciones las microempresas, a nivel del país ha mostrado durante los últimos años un crecimiento sostenido, fruto del esfuerzo del empresariado, el cual viene generando inversión y trabajo en el país. Sin embargo, todavía no alcanza el nivel de competitividad necesaria para la exportación de sus productos y enfrentar sobre todo los retos que demandan la firma del Tratado de Libre Comercio (TLC). Es imperante que ante estos nuevos retos de la globalización de los mercados, las microempresas del sector confecciones estén preparados no sólo económicamente sino culturalmente, puesto que estos nuevos retos competitivos requiere de empresarios capaces de vencer los paradigmas organizacionales existentes, capaces de asumir riesgos y vencer la resistencia al cambio.

En el distrito de Tacna, se puede distinguir el conglomerado de microempresas del sector confecciones textiles, las que se encuentran ubicadas en el Centro Comercial Tacna Centro, Coronel Mendoza y Patricio Meléndez; cuyas actividades principales es la confección y comercialización de prendas de vestir, tales como ropa deportiva, uniformes, ropa de vestir, etc.

En la actualidad los microempresarios del sector confecciones de los mencionados sectores del distrito de Tacna, no son conscientes de los desafíos que exige la globalización, puesto que en el desarrollo de sus actividades comerciales observan conductas internas que impiden 0 restringen el desarrollo de sus actividades. Se observa además que sólo actúan bajo palabra ante el compromiso de la asociatividad empresarial, pero que en la práctica muestran mayor celo y desconfianza, mostrando actitudes de renuencia y desconfianza, ante los retos que demandan la asociatividad. Se observa además que el equipamiento e infraestructura no son las más adecuadas para el desarrollo de sus actividades; sus directivos no tienen una mayor preparación en el campo empresarial; más bien han sido formados empíricamente. Estos hechos y actitudes no permiten que logren mayor emprendimiento y despegue en las actividades desarrolladas en el sector confecciones en el distrito Tacna, período 2012.

\section{Materiales y métodos}

El tipo de investigación es descriptivo correlacionar; trabaja sobre realidades de hecho y su característica fundamental es la de presentar una interpretación correcta, tiende a la descripción de las variables y determinar la relación entre las mismas (cultura organizacional y la asociatividad empresarial de las microempresas del sector confecciones de Tacna).

El diseño, es no experimental, transaccional, por que el investigador no interviene en el hecho investigado, se han recogido los datos en un determinado momento (2012).

Las unidades de estudio la constituyen 135 empresas del sector confecciones textiles formalmente constituidas en el distrito de Tacna.

La muestra corresponde al tipo aleatorio probabilístico; el muestreo probabilístico es una técnica de muestreo en virtud de la cual las muestras son recogidas en un proceso que brinda a todos los individuos de la población las mismas oportunidades de ser seleccionados.

Para la recolección de datos se recurrió al cuestionario y la entrevista como instrumentos de medición, las que fueron aplicadas a los representantes de las microempresas del sector confecciones textiles del distrito de Tacna. Los resultados son analizados, interpretados y comprobados empleando para ello los Software Estadísticos SPSS y Excel.

Desde el punto de vista geográfico el presente trabajo de investigación es a nivel Tacna, por que tratará acerca del instituto procesal de la Terminación Anticipada como mecanismo de simplificación procesal orientada una justicia célere y eficiente en el Distrito Judicial de Tacna, 2009-2013. 


\section{Resultados: Cultura Organizacional}

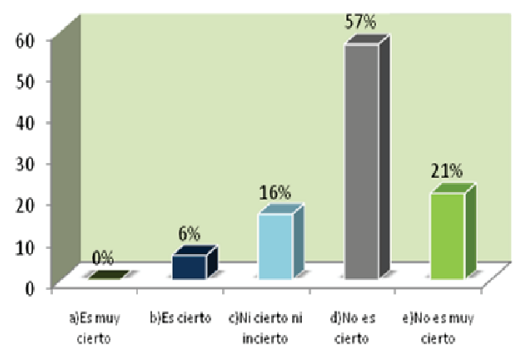

Figura 1

Nos gusta formar parte de un equipo y que nuestro rendimiento se evalúe en términos de mi contribución al equipo.

El $57 \%$ de los microempresarios manifiestan que "no es cierto" que les gusta formar parte de un equipo y que el rendimiento se evalúe en términos de la contribución al equipo; y, sólo el $6 \%$ manifiesta lo contrario. Las actitudes asumidas son productos de los intereses personales que manifiestan los trabajadores, quienes realizan sus actividades en función de sus necesidades personales. (Figura1).

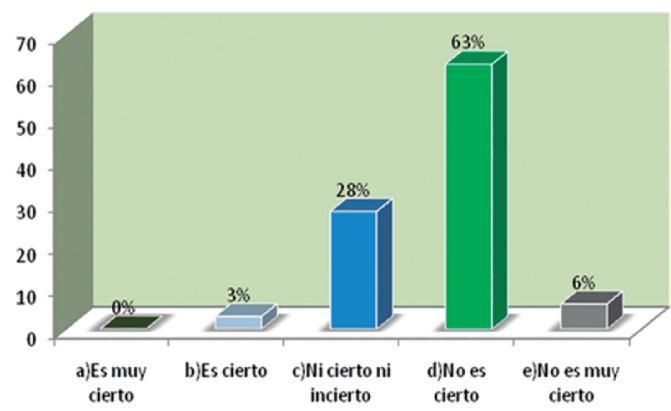

Figura 2

Fácilmente podemos ponernos de acuerdo.

El $63 \%$ de los encuestados manifiestan que "no es cierto" que, fácilmente se ponen de acuerdo; y, sólo el 3\% manifiesta lo contrario. Los miembros de la organización evidencian con los resultados obtenidos la falta de consenso entre ellos, prefieren más bien optar por actitudes individualistas y de desconfianza. (Figura2).

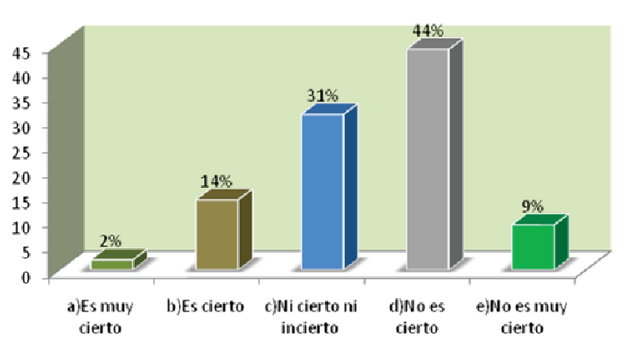

Figura 3

Siempre estamos dispuestos a asumir riesgos.

El $44 \%$ de los encuestados manifiestan que "no es cierto" que, siempre están dispuestos a asumir riesgos; y, el 2\% manifiesta lo contrario. Las declaraciones vertidas son producto de la desconfianza y el temor imperante en el medio. (Figura 3).

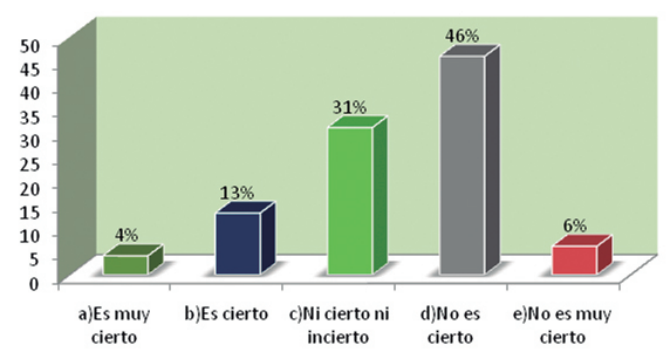

Figura 4

La empresa respalda todos los proyectos innovadores aunque estos signifiquen un alto riesgo organizacional

El $46 \%$ de los encuestados, manifiestan que "no es cierto" que la empresa respalda todos los proyectos innovadores aunque estos signifiquen un alto riesgo organizacional; y, sólo el $4 \%$ señala que es "muy cierto" que la empresa lo considere. Estos resultados nos demuestran que las microempresas no están en función de la economía globalizada, la cual exige competitividad a través de la innovación.. (Figura 4). 


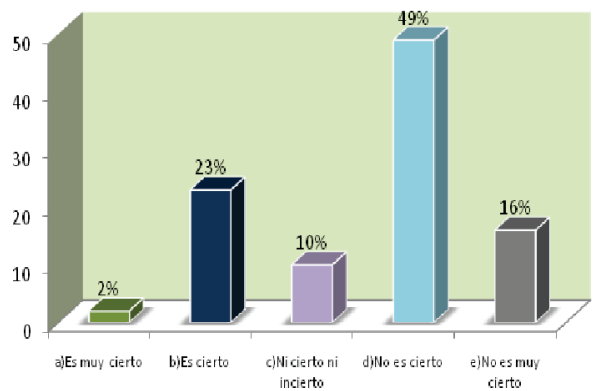

Figura 5

La empresa mantiene una política de incentivos para todos aquellos que se involucren en proyectos de innovación

El $49 \%$ de los encuestados consideran que "no es cierto" que la empresa mantiene una política de incentivos para todos aquellos que se involucren en proyectos de innovación; y, sólo el $2 \%$ manifiesta lo contrario. Los resultados evidencia la ausencia de medidas motivadoras que permitan la satisfacción laboral de los trabajadores. (Figura 5).

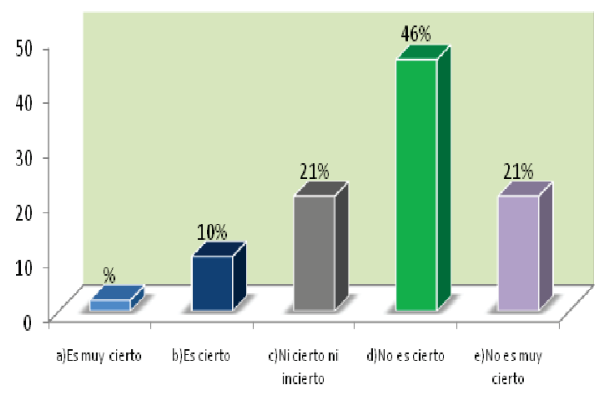

Figura 6

La empresa apuesta por comercializar productos innovadores para ser competitivos.

El $46 \%$ de los encuestados manifiestan que es "no es cierto" que la empresa apuesta por comercializar productos innovadores para ser competitivos; y; sólo el $2 \%$ señala lo contrario. Las microempresas del sector trabajan en función de precios bajos, producto de la competencia en el sector, pocas veces asumen una política innovadora como lineamiento de acción empresarial, ya que perciben este tipo de estrategia como gasto y no como inversión.(Figura 6)

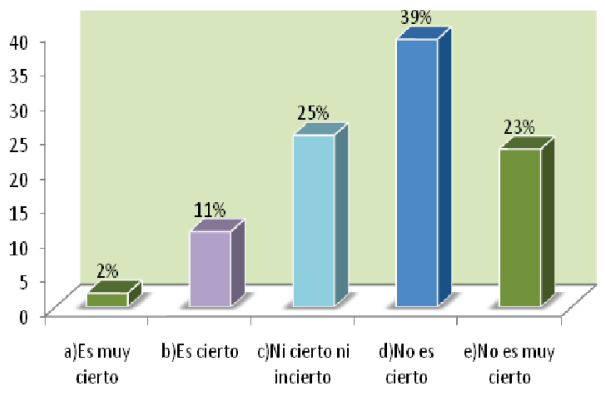

Figura 7

La empresa propicia que el personal trate abiertamente las discrepancias, críticas $y$ enfrentamientos.

El $39 \%$ de los encuestados manifiestan que "no es cierto" que la empresa propicia que el personal trate abiertamente las discrepancias, críticas y enfrentamientos; $y$, sólo el $2 \%$ señala lo contrario. Las microempresas realizan actividades empíricamente, no consideran medidas que permita la integración entre sus miembros. (Figura 7).

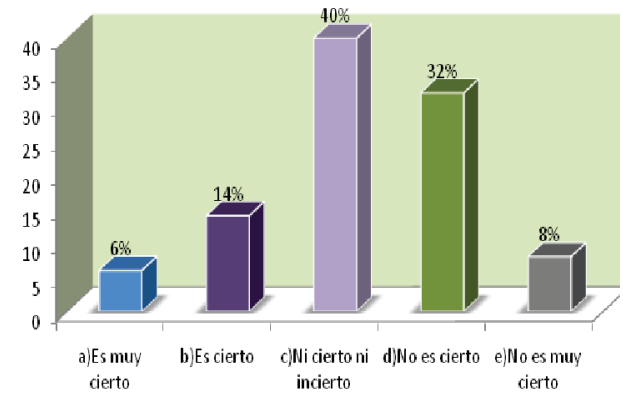

Figura 8

Muchos de los proyectos iniciados son acabados por el respaldo de la dirección.

El $40 \%$ de los microempresarios encuestados señalan que "ni cierto ni incierto" que muchos de los proyectos iniciados son acabados por el respaldo de la dirección; y, sólo el $6 \%$ señala lo contrario. Las microempresas suelen en muchas ocasiones dar preferencia a las actividades de mayor resultado económico, dejándose de lado proyectos de menor utilidad para la empresa. (Figura 8). 


\section{Resultados: Asociatividad Empresarial}

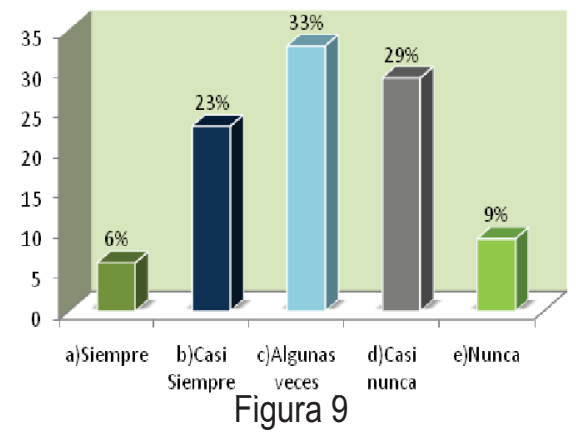

En la empresa se fabrican productos por encargo de otras organizaciones.

El $39 \%$ de los encuestados manifiestan que "algunas veces", en la empresa se fabrican productos por encargo de otras organizaciones; y, sólo el $6 \%$ señala que "nunca" lo considera. Estas aseveraciones son producto del incremento en la demanda del sector, ya que los productos no solo son demandados por el mercado local sino también por el internacional. (Figura 9).

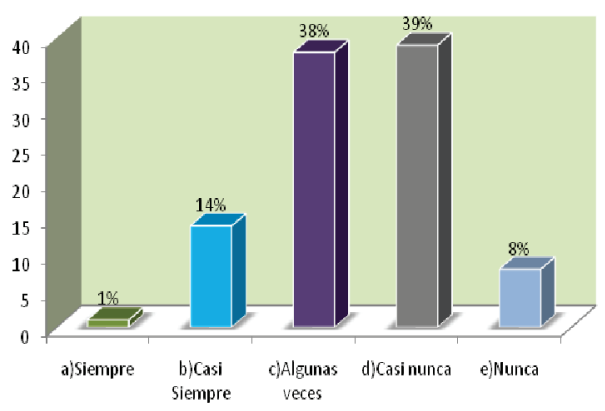

Figura 10

La empresa encarga a otra organización la fabricación de productos que luego son comercializadas como propias.

El $49 \%$ de los encuestados consideran que "casi nunca" la empresa encarga a otra organización la fabricación de productos que luego son comercializadas como propias; y, sólo el 1\% señala que "siempre" lo hacen. Las microempresas no han optado por dichas medidas, ya que prefieren comercializar sus productos a menor escala. (Figura 10).

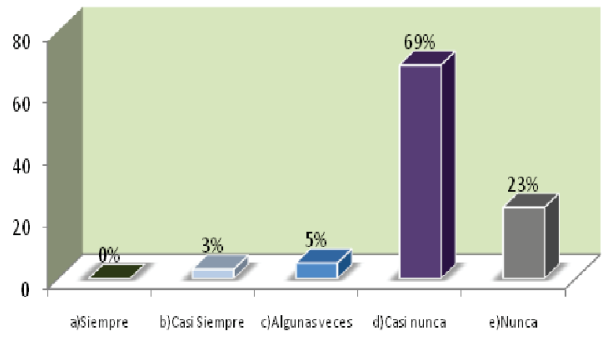

Figura 11

La empresa ha participado en la conformación de Consorcios para realizar actividades de interés conjunto para las organizaciones del sector confecciones textiles.

El $69 \%$ de los encuestados consideran que "casi nunca" la empresa ha participado en la conformación de Consorcios para realizar actividades de interés conjunto para las organizaciones del sector confecciones textiles; y, sólo el 3\% manifiesta lo contrario. (Figura11).

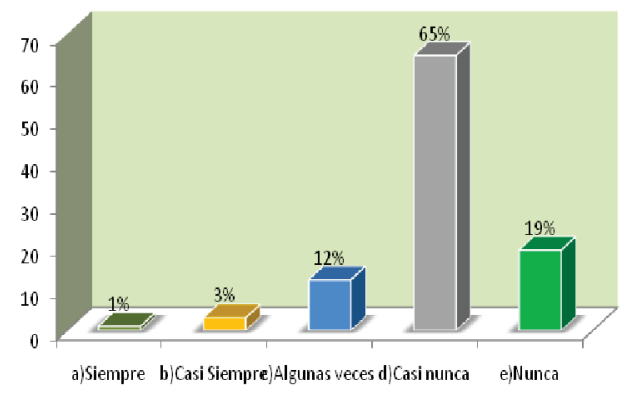

Figura 12

Se han celebrado contratos a largo plazo para la obtención de materia prima.

El $65 \%$ de los encuestados señalan que "casi nunca" se han celebrado contratos a largo plazo para la obtención de materia prima; y, sólo el $1 \%$ señala que "siempre" se considera. La nula predisposición hacia la asociatividad no permite a las microempresas tener una mayor visión y proyección hacia actividades que les permita ampliar su panorama empresarial. (Figura12). 


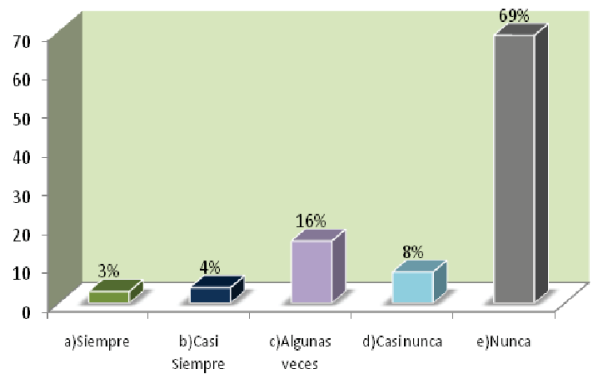

Figura 13

Se han adquirido franquicias de otros negocios como estrategia de crecimiento.

El $69 \%$ de los encuestados consideran que "nunca", se han adquirido franquicias de otros negocios como estrategia de crecimiento; $y$, sólo el $3 \%$ señala lo contrario. Las manifestaciones son a consecuencia de que las microempresas no apuestan por este tipo de estrategia de asociatividad para realizar sus actividades empresariales y reducir el esfuerzo financiero que exige cualquier estrategia de expansión. (Figura 13).

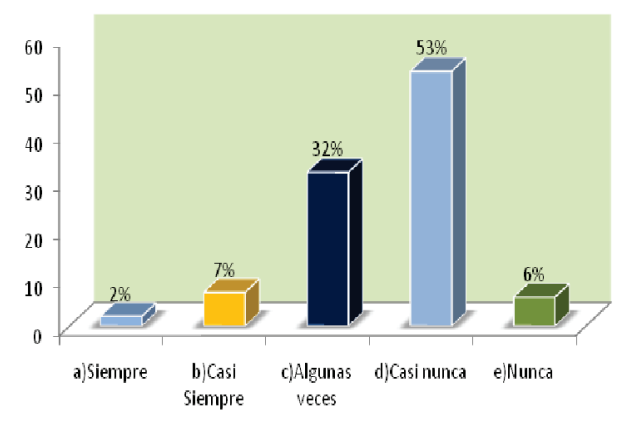

Figura 14

La empresa participa en actividades para la difusión de información sobre sus productos dirigidos a organizaciones de otros sectores.

El $65 \%$ de los encuestados consideran que "casi nunca" la empresa participa en actividades para la difusión de información sobre sus productos dirigidos a organizaciones de otros sectores; $y$, sólo el $2 \%$ señala lo contrario. Algunas de las microempresas han considerado este tipo de estrategia, con la finalidad de expandirse y obtener un mayor porcentaje de cobertura en el mercado. (Figura 14).

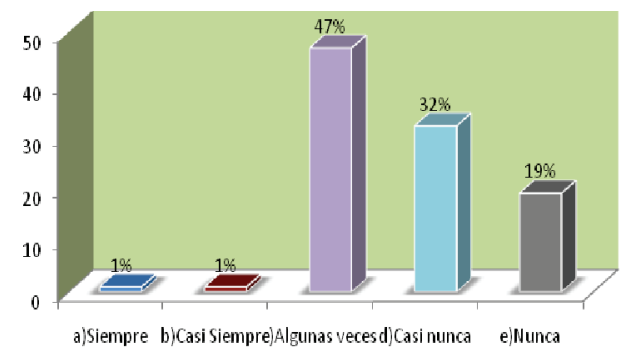

Figura 15

La empresa adopta como política de negocios la diversificación de sus productos.

El $47 \%$ de los empresarios encuestados señalan que "algunas veces" la empresa adopta como política de negocios la diversificación de sus productos; el $1 \%$ manifiesta que "siempre" lo considera. Las microempresas suelen realizar sus operaciones comerciales en función de los requerimientos de la demanda, teniendo en cuenta sobre todo lo que es de mayor beneficio para ellos.(Figura 15).

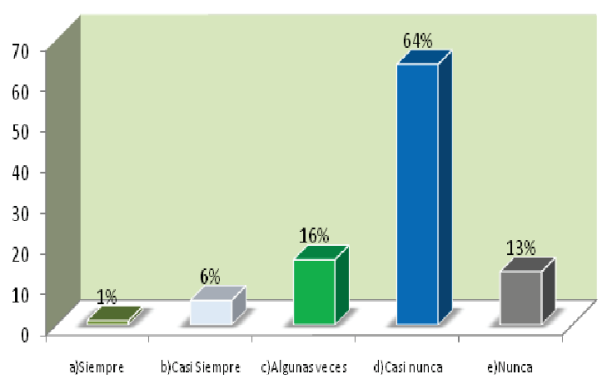

Figura 16

La empresa apuesta por la comercialización de productos innovadores para lograr la competitividad en el mercado.

El $64 \%$ de los encuestados señalan que "casi nunca" la empresa apuesta por la comercialización de productos innovadores para lograr la competitividad en el mercado; y, el 1\% manifestó que "siempre" lo considera. Las microempresas se hallan enfrascadas en la confección y comercialización de productos únicos, ellos asumen que se han especializado en ellos y que por ende no necesitan ampliar su cartera de productos. (Figura 16) 


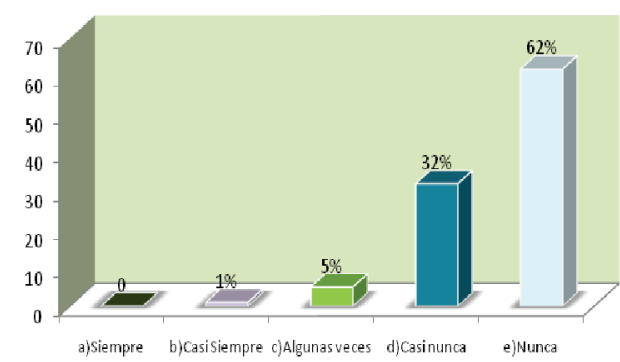

Figura 17

La empresa ha firmado contratos para la utilización de tecnología desarrollada por otras empresas.

El $62 \%$ de los encuestados manifiestan que "nunca" la empresa ha firmado contratos para la utilización de tecnología desarrollada por otras empresas; y, sólo el $1 \%$ señala que "casi siempre" lo han considerado. Estas afirmaciones se deben a que las microempresas no consideran este tipo de estrategias de competitividad para el desarrollo de sus actividades comerciales, ya que perciben que sólo les hará incurrir en mayores gastos que perjudican su rentabilidad. (Figura 17).

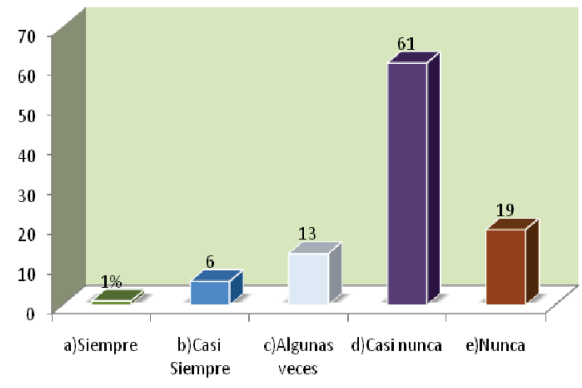

Figura 18

La empresa ha firmado contratos para la comercialización de productos.

El $61 \%$ de los encuestados consideran que "casi nunca" la empresa ha firmado contratos para la comercialización de productos; y, sólo el 1\% señalan que "siempre" lo considera. La mayoría de las microempresas producen en menores porcentajes, para poder comercializar en mayor escala requieren asociarse para alcanzar mejores y rentables resultados económicos. (Figura 18).

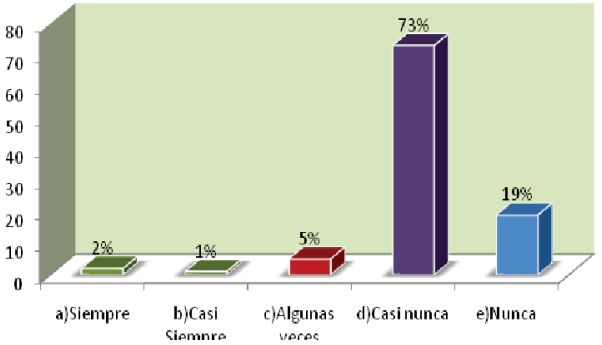

Figura 19

La empresa considera la definición de indicadores para evaluar la eficacia de las alternativas para la diversificación de productos.

El $73 \%$ de los encuestados manifiestan que "casi nunca" la empresa considera la definición de indicadores para evaluar la eficacia de las alternativas para la diversificación de productos; y, sólo el $2 \%$ señala que "siempre" lo considera. La falta de capacitación en gestión y competitividad empresarial, no les permite a las microempresas considerar este tipo de actividades para el mejoramiento de su desempeño organizacional. (Figura 19).

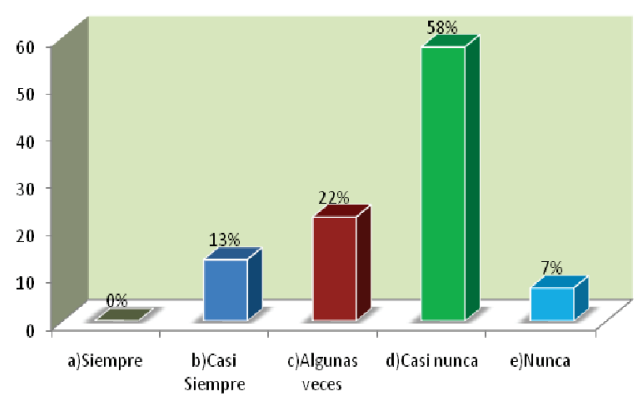

Figura 20

La empresa ha celebrado contratos con otras empresas del sector confecciones para la distribución conjunta de productos.

El $58 \%$ de los encuestados señalan que "casi nunca" la empresa ha celebrado contratos con otras empresas del sector confecciones para la distribución conjunta de productos; $y$, sólo el $13 \%$ manifiesta lo contrario. La cultura individualista que caracteriza al sector confecciones no les permite poder satisfacer las necesidades de la cada vez más exigente demanda. (Figura 20). 


\section{Discusión}

Los resultados de los instrumentos de medición del cuestionario aplicado a los microempresarios del sector confecciones textiles del distrito de Tacna, permitieron evaluar la cultura organizacional que presentan las microempresas del Sector Confecciones Textiles del distrito de Tacna; los resultados obtenidos nos permitieron conocer las inadecuada cultura organizacional y la deficiente asociatividad empresarial que presentan de las microempresas del Sector Confecciones Textiles del distrito de Tacna, 2012.

Las microempresas, constituyen el eje fundamental para diversificar y ampliar la oferta exportable en el sector de confecciones, por ello se hace necesario establecer mecanismos de estimulación de las microempresas que permitan aumentar su capacidad productiva; también son en el mediano plazo las encargadas de ofrecer empleo a personas de nivel medio y bajo, que constituyen el grueso del desempleo. "Sin embargo, adolecen de problemas estructurales determinados por factores internos como, la baja calificación de recurso humano, las limitaciones tecnológicas y de acceso a bienes de capital, el limitado acceso a los recursos financieros, baja calidad de gestión empresarial y reducida inserción en los mercados entre otros".

Pese a la gran variedad de productos textiles que posee nuestro país y a las posibilidades de transformación que se derivan de estos, las oportunidades de las microempresas, en el contexto económico actual no son las mejores, si bien es cierto las pequeñas y medianas empresas dedicadas a la producción y comercialización de confecciones han logrado posesionarse en el mercado nacional respondiendo a una demanda natural, el verdadero reto está en los mercados internacionales donde su presencia es reducida.

En congruencia Antonio, Guillén (2007), en el estudio: "Las redes empresariales como una alternativa para las pequeñas y medianas empresas del sector plástico en el Estado Lara", precisa que, las características identificadas para la totalidad de las organizaciones estudiadas muestran que poseen estructuras centralizadas donde la autoridad se concentra en los niveles estratégicos, con debilidad en los procesos de delegación y empoderamiento; esto se evidencia en la toma de decisiones, en la comunicación $y$, en algunos casos, en la manera como se llevan a cabo los procesos de supervisión. Al respecto, destaca cómo la toma de decisiones es centralizada $y$, aunque posee una comunicación formal con predominio de información escrita, en la supervisión y retroalimentación poco se toma en cuenta al personal. Por otro lado, en la toma de decisiones la información del cliente es determinante para establecer y definir los lineamientos estratégicos que guiarán las acciones productivas. Por esa razón puede asegurarse que existen alianzas únicamente con los clientes.

Adicionalmente, Silvina, Carranza Bigotti (2003), en el estudio de investigación titulado: Análisis de Competitividad, Productividad y Percepción de Valor de la Gran Industria Peruana Exportadora de confecciones: Alcance Comparativo con la Gran Industria Mexicana, afirma que, la industria de confecciones peruana carece de un posicionamiento definido y que luego del análisis sobre los factores realizado comparativamente con la industria mexicana es recomendable en el corto plazo aplicar una estrategia de liderazgo en costos. Sin embargo, dados los cambios predecibles en el escenario de la oferta mundial es recomendable migrar en un siguiente estadio hacia a una estrategia de enfoque, tomando en cuenta que se han encontrado segmentos de mercado no atendidos en prendas de alto valor.

Así también, Suárez, R. (2007), en el estudio de investigación: Análisis del potencial de Exportación de Confecciones de Algodón en Gamarra, dice que, Gamarra no está en capacidad de alcanzar los objetivos de exportación. Por otro lado, los lineamientos estratégicos genéricos que se proponen en este trabajo, son los siguientes: un enfoque de diferenciación en nichos de mercado que demanden productos de alta calidad, especializados y sofisticados; lo cual se logrará mediante la producción de prendas de vestir que cumplan dichas características, apoyados en un sistema de respuesta rápida.

Asimismo, como una alternativa para superar las limitaciones de exportación de Gamarra, y en concordancia con los lineamientos estratégicos, se plantea una reestructuración 
industrial del sector, mediante el modelo de un Clúster, cuyo funcionamiento está basado en la cooperación entre sus empresas, que se apoya mediante un rápido y fluido movimiento de información, una visión compartida entre las empresas y el gobierno; siendo la intervención de este último, fundamental para el éxito del Clúster.

La asociatividad empresarial es una estrategia para enfrentar los mercados globalizados y la creciente y fuerte competencia de otras empresas (nacionales y/o extranjeras). Es un mecanismo de cooperación por el cual se establecen relaciones y/o articulaciones entre empresas y/o grupos de empresas tras un objetivo común (Chávez, S.2008).

Una experiencia modelo en el Perú, es sin duda en la actualidad, el emporio comercial de Gamarra cuenta con más de 150 galerías, en un espacio de 40 cuadras, unos 14 mil locales se dedican al comercio o industria de la confección, logrando generar empleo a cerca de 60 mil personas.

Las confecciones peruanas han llegado a un total de 82 destinos. En los últimos años, estas han ingresado a países como Haití, República de Corea, Lituania, Surinam, Indonesia, Ucrania, Guyana, Rumania, Gabón, Qatar, entre otros.(Mass.2012).

Asimismo, la exportación de confecciones peruanas sumó US\$ 472.7 millones, $14 \%$ más respecto al mismo período. Estos indicadores deben ser tomados en cuenta por las mypes del sector confecciones textiles del distrito de Tacna; para ello es necesario que rompan el paradigma del individualismo y desconfianza y de esta manera sumar sinergias para poder ofrecer mejores productos en cuanto a calidad, precios, innovación y de esta manera elevar sus utilidades y su proyección en el mercado local e internacional.

Las microempresa del sector confecciones de Tacna, deben mejorar su cultura organizacional, deben poner atención en los siguientes aspectos: énfasis en el equipo, invitación y aceptación al riesgo, tolerancia al conflicto y orientación hacia los resultados, para ello deben mostrar preocupación por las formas de actuación ante los problemas y oportunidades de gestión y adaptación a los cambios y requerimientos de orden exterior e interior a fin de vencer los paradigmas organizacionales del individualismo y oportunismo, que les impide alcanzar una Cultura emprendedora que les permita aprovechar oportunidades y mejorar los niveles de competitividad en el sector de confecciones.

Así también, las microempresas del sector confecciones, deben adecuarse al impacto y a la intensidad de la velocidad de cómo están acelerando los cambios fugazmente. Para tal efecto, deberá demostrar una capacidad de respuesta capaz de absorber la incertidumbre que dichos cambios puedan ocasionar para así neutralizar o minimizar los riesgos que ponga en peligro su existencia. Se recomienda además, implementar procesos de capacitación gerencial, a fin de desarrollar competencias conducentes a la formulación de estrategias colectivas a los niveles funcional, de negocio y corporativa; fortaleciendo relaciones conjuntas, no solamente con los clientes sino también con los proveedores y competidores.

Se sugiere también el aprovechamiento de la oportunidad que les ofrece las condiciones del mercado, para ello se recomienda a los empresarios potenciar condiciones necesarias para el desarrollo de estrategias de cooperación, de tipo de integración de estándares, investigación conjunta, inversión e innovación, que les permita operar en nuevos mercados, fortalecer su posición competitiva, diversificar sus productos y las adquisiciones conjuntas.

Las microempresas del sector confecciones textiles a fin de enfrentar los mercados globalizados y la creciente y fuerte competencia de otras empresas (nacionales y/o extranjeras) requieren de análisis y preparación y apostar por la asociatividad empresarial; para ello además deben considerar como reto 10 siguiente:

- Visión: Convertirse en una potencial industria manufacturera en el sector confecciones textiles del Perú.

- Misión: unir esfuerzos individuales y potenciar la actuación conjunta de los empresarios de las microempresas del sector confecciones. 
- Función: ejecutar la articulación productiva segmentada, en el ámbito local / externo.

Las microempresas del sector confecciones textiles del distrito de Tacna a fin de poder incrementar sus ventas no sólo nacional sino internacionalmente, deben apostar por los clusters como estrategia de internacionalización para ello deben considerar lo siguiente:

- Erradicar sus paradigmas organizacionales del individualismo, desconfianza y oportunismo.

- Basar sus relaciones en la confianza, solidaridad, colaboración, compromiso y responsabilidad.

- Las empresas deberán abogar por políticas que mejoren la competitividad nacional 0 regional, como son las inversiones para crear nuevos factores y las ayudas para conseguir acceso a los mercados internacionales.

- Estructurar la iniciativa en cuatro grandes fases: la identificación y priorización de clusters, la realización de estudiosdiagnóstico sobre cada "clúster", la definición de sus planes conjuntos de mejora de la competitividad $y$, finalmente, el apoyo al desarrollo y ejecución de los planes elaborados para los clusters.

Si bien es cierto que la microempresas del sector confecciones textiles dinamizan el crecimiento económico del distrito, ya sea fomentando el empleo y/o generando nuevos recursos al distrito de Tacna, estas deben deben apostar por la asociatividad a fin de poder satisfacer la demanda que impera en el sector, todo vez que se tiene conocimiento que en el distrito de Tacna, existe un mayor porcentaje de flujo de visitantes extranjeros a quienes les resulta atractivo la oferta de productos textiles que se comercializa en Tacna.

\section{Referencias Bibliográficas}

- Guillén, A. (2007). Las redes empresariales como una alternativa para las pequeñas y medianas empresas del sector plástico en el Estado Lara. Maestría en Gestión Empresarial. Universidad Central Lisandro Alvarado. Venezuela.

- Carranza, S. (2003). Análisis de Competitividad, Productividad y Percepción de Valor de la Gran Industria Peruana Exportadora de confecciones. Maestría en Administración. ESAN. Lima, Perú.

- Suárez, R. (2007), Análisis del potencial de Exportación de Confecciones de Algodón en Gamarra, Grado de Maestría en Administración. ESAN. Lima, Perú.

- Chávez, S. (2008). Capacitación de Productores en los Beneficios de la Asociatividad. Mincetur. Perú.

- Mass (2012). Exportación de confecciones. Consultado en Junio 2013. http://mass.pe/noticias/2012/06/exportacion -de-confecciones-sumo-us4727-millones]

Email: renpis@hotmail.com

Recibido: 15/11/2014

Aceptado para publicación: $12 / 12 / 2014$ 